\title{
A shocking ice structure
}

Nature 569, 251-255 (2019)

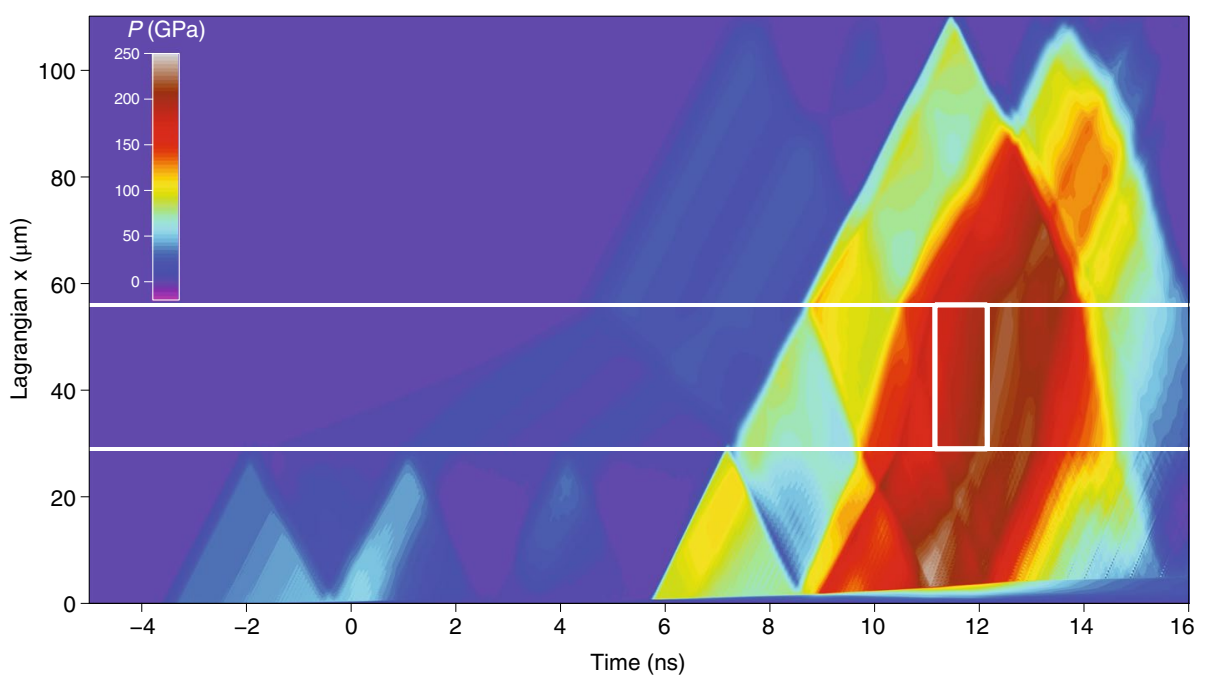

Credit: Springer Nature Ltd

Water ice is staggeringly polymorphic, with 17 unique crystalline forms (plus several amorphous ones). And now Marius Millot, Federica Coppari and co-workers take the tally up to 18 . In their proposed ice XVIII structure, the oxygens form a face-centred cubic structure. Moreover, the ionic conductivity in this solid phase of water jumps to a level more akin to metallic conductivity. This kind of 'superionic' behaviour occurs when protons (of the hydrogen atoms) are able to move through the crystal as a liquid.

The experiment involves six highpower laser beams firing in sequence to create compression shockwaves (pictured: a simulation of the Lagrangian, which is a measure of longitudinal stress, as a function of time) within a thin layer of water held between two diamond layers. In this setup, the water reaches temperatures of $2,000-3,000$ kelvin and pressures of 100-420 gigapascals $(\mathrm{GPa})$ within nanoseconds. Simultaneous X-ray diffraction (white box marking $11 \mathrm{~ns}$ ) confirms the structure of the 4-7 nm-sized ice crystals.

Superionic water ice above $100 \mathrm{GPa}$ was first predicted in 1988. Such conditions are believed to exist within the interiors of the ice giants, Neptune and Uranus, $65 \%$ of which could be water. Millot et al. conclude that their results support estimates that superionic ice may be stable $8,000 \mathrm{~km}$ below the surface (or one-third of the radius of Neptune and Uranus). The superionic ice flows within the ice giants would resemble the slow convection within Earth's mantle (rather than the rapidly swirling liquid iron of the outer core).

\section{May Chiao}

Published online: 31 May 2019

https://doi.org/10.1038/s41550-019-0825-9 